\title{
Free vibration analysis of beams by using a third-order shear deformation theory
}

\author{
MESUT ŞIMMŞEK and TURGUT KOCATÜRK \\ Department of Civil Engineering, Yildiz Technical University, Yildiz 34349, \\ Istanbul, Turkey \\ e-mail: kocaturk@yildiz.edu.tr
}

MS received 31 March 2006; revised 29 September 2006

\begin{abstract}
In this study, free vibration of beams with different boundary conditions is analysed within the framework of the third-order shear deformation theory. The boundary conditions of beams are satisfied using Lagrange multipliers. To apply the Lagrange's equations, trial functions denoting the deflections and the rotations of the cross-section of the beam are expressed in polynomial form. Using Lagrange's equations, the problem is reduced to the solution of a system of algebraic equations. The first six eigenvalues of the considered beams are calculated for different thickness-to-length ratios. The results are compared with the previous results based on Timoshenko and Euler-Bernoulli beam theories.
\end{abstract}

Keywords. Free vibrations of beams; the third-order shear deformation theory; Lagrange's equations; Lagrange multipliers.

\section{Introduction}

There are many studies on the theory and analysis of beam-type structures in the literature. The oldest and the well-known beam theory is the Euler-Bernoulli beam theory (or classical beam theory-CBT) which assumed that straight lines perpendicular to the mid-plane before bending remain straight and perpendicular to the mid-plane after bending. As a result of this assumption, transverse shear strain is neglected. Although this theory is useful for slender beams and plates, it does not give accurate solutions for thick beams and plates. The next theory is the Timoshenko beam theory (the first order shear deformation theory-FSDT) which assumed that straight lines perpendicular to the mid-plane before bending remain straight, but no longer remain perpendicular to the mid-plane after bending. In FSDT, the distribution of the transverse shear stress with respect to the thickness coordinate is assumed constant. Thus, a shear correction factor is required to compensate for the error because of this assumption in FSDT. The third-order shear deformation theory (TSDT) which assumed parabolic distribution of the transverse shear stress and strain with respect to the thickness coordinate was proposed for beams with rectangular cross-sections (Wang et al 2000). Also, zero transverse shear stress condition of the upper and lower fibres of the cross-section is satisfied without a shear correction factor in TSDT. 
There are many studies related with the problem of free vibration of beams based on CBT and FSDT (Timoshenko \& Young 1955; Hurty \& Rubinstein 1967; Farghaly 1994; Banerjee 1998; Nallim \& Grossi 1999; Kim \& Kim 2001; Lee et al 2003; Auciello \& Ercolano 2004; Zhou 2001; Lee \& Schultz 2004; Şimşek 2005a, b; Kocatürk \& Şimşek 2005a, b). The relationship between the bending solution of TSDT and those of CBT and FSDT was presented (Wang et al 2000). The exact stiffness matrix was derived from the solutions of differential equations according to TSDT for isotropic beams (Eisenberger 2003). Frequency equations and characteristic functions of homogeneous orthotropic beams having different boundary conditions were obtained, and the first six natural frequency parameter was tabulated for different values of stiffness ratios and values of thickness-to-length ratios (Soldatos \& Sophocleous 2001). Static deflections of the laminated composite beams subjected to uniformly distributed load were studied using the classical, the first-order, the second-order and the third-order beam theories (Khdeir \& Reddy 1997).

In the present study, free vibration of beams with different boundary conditions is analysed based on the third-order shear deformation theory (TSDT). Frequency equations of the beams are derived using Lagrange's equations. The boundary conditions of the beams are considered using Lagrange multipliers. The trial functions for the deflections and rotations of the cross-section of the beam are selected in polynomial form. The first six eigenvalues of the considered beams are calculated for different thickness-to-length ratios. The obtained results are compared with earlier results based on CBT and FSDT.

\section{Theory and formulations}

A straight uniform beam of length $L$, width $b$, depth $h$, having rectangular cross-section is shown in figure 1. A Cartesian coordinate system $(x, y, z)$ is defined on the central axis of the beam, where the $x$ axis is taken along the central axis, the $y$ axis in the width direction and the $z$ axis in the depth direction. Also, the origin of the coordinate system is chosen at the mid-point of the total length of the beam.

The third-order shear deformation theory (TSDT) is based on the following displacement fields (Wang et al 2000);

$$
\begin{aligned}
& u_{x}(x, z, t)=z \phi(x, t)-\alpha z^{3}\left[\phi(x, t)+w_{, x}(x, t)\right] \\
& u_{z}(x, z, t)=w(x, t),
\end{aligned}
$$

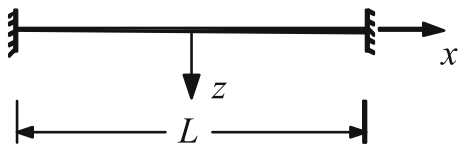

(a)

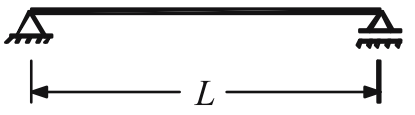

(c)

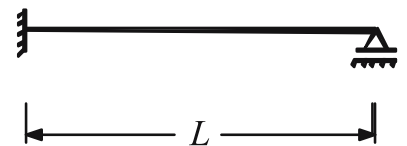

(b)

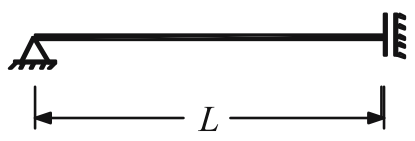

(d)

Figure 1. (a) Clamped-clamped, (b) clamped-pinned, (c) pinned-pinned, (d) pinned-guided straight uniform beams with rectangular cross-section. 
where $u_{x}$ and $u_{z}$ are displacements in $x$ and $z$ directions at any material point in the $(x, z)$ plane, $\alpha=4 /\left(3 h^{2}\right), w$ is the transverse displacements, and $\phi$ represents the slope $\partial u_{x} / \partial z$ at $z=0$ of the deformed line which was straight in the undeformed beam. In this case $\phi(x, t)$ and $\alpha$ together define the third-order nature of the deformed line. The symbol ()$_{, x}$ indicates the derivative with respect to $x$. The strain-displacement relations are given by

$$
\begin{aligned}
& \varepsilon_{x x}=u_{x, x}=z \phi_{, x}-\alpha z^{3}\left(\phi_{, x}+w_{, x x}\right), \\
& \gamma_{x z}=u_{x, z}+u_{z, x}=\left(1-3 \alpha z^{2}\right)\left(\phi+w_{, x}\right) .
\end{aligned}
$$

The constitutive relations between stresses and strains for the linear elastic material become

$$
\sigma_{x x}=E \varepsilon_{x x} ; \quad \tau_{x z}=G \gamma_{x z},
$$

where $\sigma_{x x}$ is the longitudinal normal stress, $\varepsilon_{x x}$ the longitudinal normal strain, $\tau_{x z}$ the transverse shear stress, $\gamma_{x z}$ the transverse shear strain, $E$ the Young's modulus, and $G$ the shear modulus. The strain energy of the beam in Cartesian coordinates is

$$
V=\frac{1}{2} \int_{-L / 2}^{L / 2} \int_{A}\left(\sigma_{x x} \varepsilon_{x x}+\tau_{x z} \gamma_{x z}\right) \mathrm{d} A \mathrm{~d} x .
$$

Using (2), (3) and (4), the strain energy of the beam at any instant can be expressed as:

$$
\begin{aligned}
V= & \frac{1}{2} \int_{-L / 2}^{L / 2}\left\{D_{x x}\left(\phi_{, x}\right)^{2}-2 \alpha F_{x x} \phi_{, x}\left(\phi_{, x}+w_{, x x}\right)\right. \\
& +\alpha^{2} H_{x x}\left[\left(\phi_{, x}\right)^{2}+2 \phi_{, x} w_{, x x}+\left(w_{, x x}\right)^{2}\right] \\
& \left.+\left(A_{x z}-6 \alpha D_{x z}+9 \alpha^{2} F_{x z}\right)\left[\phi^{2}+2 \phi w_{, x}+\left(w_{, x}\right)^{2}\right]\right\} \mathrm{d} x,
\end{aligned}
$$

where

$$
\left(D_{x x}, F_{x x}, H_{x x}\right)=\int_{A}\left(z^{2}, z^{4}, z^{6}\right) E \mathrm{~d} A, \quad\left(A_{x z}, D_{x z}, F_{x z}\right)=\int_{A}\left(1, z^{2}, z^{4}\right) G \mathrm{~d} A .
$$

It follows from (1) that the velocities of any point on the beam take the form;

$$
v_{x}=\dot{u}_{x}(x, z, t)=z \dot{\phi}-\alpha z^{3}\left(\dot{\phi}+\dot{w}_{, x}\right), \quad v_{z}=\dot{u}_{z}(x, z, t)=\dot{w} .
$$

The kinetic energy of the beam at any instant is

$$
T=\frac{1}{2} \int_{-L / 2}^{L / 2} \int_{A} \rho\left(v_{x}^{2}+v_{z}^{2}\right) \mathrm{d} A \mathrm{~d} x,
$$

where $\rho$ is the mass of the beam per unit volume. By defining the following cross-sectional inertial coefficients

$$
\left(I_{A}, I_{D}, I_{F}, I_{H}\right)=\int_{A}\left(1, z^{2}, z^{4}, z^{6}\right) \rho \mathrm{d} A
$$


and after some algebraic manipulations, the kinetic energy of the beam at any instant is obtained in the following form;

$$
\begin{aligned}
T= & \frac{1}{2} \int_{-L / 2}^{L / 2}\left\{I_{A}(\dot{w})^{2}+I_{D}(\dot{\phi})^{2}-2 \alpha I_{F} \dot{\phi}\left(\dot{\phi}+\dot{w}_{, x}\right)\right. \\
& \left.+\alpha^{2} I_{H}\left[(\dot{\phi})^{2}+2 \dot{\phi} \dot{w}_{, x}+\left(\dot{w}_{, x}\right)^{2}\right]\right\} \mathrm{d} x .
\end{aligned}
$$

In order to apply Lagrange's equations, the trial functions $w(x, t)$ and $\phi(x, t)$ are approximated by space-dependent polynomial terms $x^{0}, x^{1}, x^{2}, \ldots, x^{N-1}$ and time-dependent generalized coordinates $a_{n}(t)$ and $b_{n}(t)$. Therefore, by using Lagrange's equations, by assuming the displacement $w(x, t)$ and the rotation of cross-sections $\phi(x, t)$ to be representable by a series of admissible functions and adjusting the coefficients in the series to satisfy Lagrange's equations, approximate solutions are found for the displacement and rotation functions. Thus;

$$
\begin{aligned}
& w(x, t)=\sum_{n=1}^{N} a_{n}(t) x^{n-1}, \\
& \phi(x, t)=\sum_{n=1}^{N} b_{n}(t) x^{n-1} .
\end{aligned}
$$

The constraint conditions of the supports are satisfied using the Lagrange multipliers. It should be noted at this stage that while both CBT and FSDT have two boundary conditions at each support of the beam, TSDT has three at each support. Essential and natural boundary conditions for TSDT theory are given below (Wang et al 2000):

The essential (kinematic or geometric) boundary conditions:

$$
w, w_{, x}, \phi
$$

The natural (dynamic) boundary conditions:

$$
\widehat{V}_{x}=\alpha \frac{\mathrm{d} P_{x x}}{\mathrm{~d} x}+\widehat{Q}_{x}, \alpha P_{x x}, \widehat{M}_{x x},
$$

where $\widehat{V}_{x}$ is the effective shear force, and the quantities $\widehat{M}_{x x}, P_{x x}, \widehat{Q}_{x}$ are defined as follows (Wang et al 2000):

$$
\widehat{M}_{x x}=M_{x x}-\alpha P_{x x}, \widehat{Q}_{x x}=Q_{x}-3 \alpha R_{x},
$$

where

$$
M_{x x}=\int_{A} z \sigma_{x x} \mathrm{~d} A, \quad P_{x x}=\int_{A} z^{3} \sigma_{x x} \mathrm{~d} A, \quad Q_{x}=\int_{A} \tau_{x z} \mathrm{~d} A, \quad R_{x}=\int_{A} z^{2} \tau_{x z} \mathrm{~d} A .
$$

It is known that some expressions satisfying essential (geometric) boundary conditions are chosen for $w(x, t), \phi(x, t)$, and by using the Lagrange's equations, the natural boundary conditions are also satisfied. Therefore, by choosing the appropriate boundary conditions given by (12) and (13), the constraint conditions of the beams are given as follows: 
(i) For the clamped-clamped beam

$$
\begin{aligned}
w\left(x_{A}, t\right) & =0, w_{, x}\left(x_{A}, t\right)=0, \phi\left(x_{A}, t\right)=0, w\left(x_{B}, t\right)=0, \\
w_{, x}\left(x_{B}, t\right) & =0, \phi\left(x_{B}, t\right)=0 .
\end{aligned}
$$

(ii) For the clamped-pinned beam

$$
w\left(x_{A}, t\right)=0, w_{, x}\left(x_{A}, t\right)=0, \phi\left(x_{A}, t\right)=0, w\left(x_{B}, t\right)=0 .
$$

(iii) For the pinned-guided beam

$$
w\left(x_{A}, t\right)=0, w_{, x}\left(x_{B}, t\right)=0, \phi\left(x_{B}, t\right)=0 .
$$

(iv) For the pinned-pinned beam

$$
w\left(x_{A}, t\right)=0, w\left(x_{B}, t\right)=0 .
$$

$x_{A}$ and $x_{B}$ denote the location of left and right supports of the beam respectively. By introducing the Lagrange multipliers formulation, the Lagrangian functional of the problem is obtained as follows:

$$
J=T-V+G_{S},
$$

where for the clamped-clamped beam

$$
\begin{aligned}
G_{S}= & \theta_{1} \cdot w\left(x_{A}, t\right)+\beta_{1} \cdot w_{, x}\left(x_{A}, t\right)+\delta_{1} \cdot \phi\left(x_{A}, t\right)+\theta_{2} \cdot w\left(x_{B}, t\right) \\
& +\beta_{2} \cdot w_{, x}\left(x_{B}, t\right)+\delta_{2} \cdot \phi\left(x_{B}, t\right)
\end{aligned}
$$

for the clamped-pinned beam

$$
G_{S}=\theta_{1} \cdot w\left(x_{A}, t\right)+\beta_{1} \cdot w_{, x}\left(x_{A}, t\right)+\delta_{1} \cdot \phi\left(x_{A}, t\right)+\theta_{2} \cdot w\left(x_{B}, t\right) ;
$$

for the pinned-guided beam

$$
G_{S}=\theta_{1} \cdot w\left(x_{A}, t\right)+\beta_{2} \cdot w_{, x}\left(x_{B}, t\right)+\delta_{2} \cdot \phi\left(x_{B}, t\right) ;
$$

for the pinned-pinned beam

$$
G_{S}=\theta_{1} \cdot w\left(x_{A}, t\right)+\theta_{2} \cdot w\left(x_{B}, t\right) .
$$

In equations (21) to (24), $\theta_{i}, \beta_{i}$ and $\delta_{i}$ are the Lagrange multipliers. The Lagrange's equations are given as follows:

$$
\frac{\partial J}{\partial q_{k}}-\frac{\mathrm{d}}{\mathrm{d} t} \frac{\partial J}{\partial \dot{q}_{k}}=0 \quad k=1,2, \ldots, 2 N+M
$$

where the overdot stands for the partial derivative with respect to time, $M$ is the number of the Lagrange multipliers, and

$$
\begin{aligned}
& q_{k}=a_{n} \quad k=1,2, \ldots, N \\
& q_{k}=b_{n-N} \quad k=N+1, \ldots, 2 N
\end{aligned}
$$


for the clamped-clamped beam

$$
q_{2 N+1}=\theta_{1}, q_{2 N+2}=\theta_{2}, q_{2 N+3}=\beta_{1}, q_{2 N+4}=\beta_{2}, q_{2 N+5}=\delta_{1}, q_{2 N+6}=\delta_{2} ;
$$

for the clamped-pinned beam

$$
q_{2 N+1}=\theta_{1}, q_{2 N+2}=\theta_{2}, q_{2 N+3}=\beta_{1}, q_{2 N+4}=\delta_{1}, q_{2 N+5}=0, q_{2 N+6}=0,
$$

for the pinned-guided beam

$$
q_{2 N+1}=\theta_{1}, q_{2 N+2}=\beta_{2}, q_{2 N+3}=\delta_{2}, q_{2 N+4}=0, q_{2 N+5}=0, q_{2 N+6}=0,
$$

for the pinned-pinned beam

$$
q_{2 N+1}=\theta_{1}, q_{2 N+2}=\theta_{2}, q_{2 N+3}=0, q_{2 N+4}=0, q_{2 N+5}=0, q_{2 N+6}=0 .
$$

For free vibration of the beam, the time-dependent generalized displacement coordinates can be expressed as follows:

$$
\begin{aligned}
& a_{n}(t)=\bar{a}_{n} \exp ^{i \omega t}, \\
& b_{n}(t)=\bar{b}_{n} \exp ^{i \omega t},
\end{aligned}
$$

where $\omega$ is the natural frequency of the beam. Dimensionless amplitudes of the displacement and normal rotation of a cross-section of the beam can be expressed as follows:

$$
\begin{aligned}
& \bar{w}(x)=\sum_{n=1}^{N} \bar{a}_{n} x^{n-1}, \\
& \bar{\phi}(x)=\sum_{n=1}^{N} \bar{b}_{n} x^{n-1} .
\end{aligned}
$$

Introducing the following non-dimensional parameters

$$
\widehat{x}=\frac{x}{L}, \widehat{w}=\frac{w}{L}, \widehat{\phi}=\phi, \quad \lambda^{2}=\frac{\rho A \omega^{2} L^{4}}{E I}, \quad \mu=\frac{h^{2}}{L^{2}}, \quad \kappa=\frac{L^{2}}{h^{2}(1+v)}
$$

and using (25), the following simultaneous sets of linear algebraic equations (frequency equation) are obtained which can be expressed in the following matrix form

$$
\tilde{K} \tilde{q}-\lambda^{2} \tilde{M} \tilde{q}=0
$$

The elements of stiffness matrix $\tilde{K}$ and the mass matrix $\tilde{M}$ are given in the Appendix. The eigenvalues (characteristic values) $\lambda$ are found from the condition that the determinant of the system of equations given by (34) must vanish.

\section{Numerical results}

The first six dimensionless frequency parameters (eigenvalues) of the beams with clampedclamped (CC), clamped-pinned (CP), pinned-pinned (PP), pinned-guided (PG) boundary 
Table 1. Convergence study of the first six dimensionless frequency parameters $\lambda_{i}$ of the pinned-pinned (PP) beam for $h / L=0 \cdot 1$ according to TSDT.

\begin{tabular}{rcccccc}
\hline$N$ & $\lambda_{1}$ & $\lambda_{2}$ & $\lambda_{3}$ & $\lambda_{4}$ & $\lambda_{5}$ & $\lambda_{6}$ \\
\hline 6 & $3 \cdot 115916$ & $6 \cdot 102613$ & $9 \cdot 829280$ & $13 \cdot 85760$ & - & - \\
8 & $3 \cdot 115696$ & $6 \cdot 090875$ & $8 \cdot 873625$ & 11.52865 & $16 \cdot 07145$ & - \\
10 & $3 \cdot 115696$ & $6 \cdot 090825$ & $8 \cdot 841825$ & 11.35213 & $13 \cdot 84615$ & $16 \cdot 36586$ \\
12 & $3 \cdot 115696$ & $6 \cdot 090825$ & $8 \cdot 841488$ & 11.34637 & $13 \cdot 63057$ & $15 \cdot 75051$ \\
14 & $3 \cdot 115696$ & $6 \cdot 090825$ & $8 \cdot 841488$ & $11 \cdot 34631$ & $13 \cdot 62094$ & 15.69566 \\
16 & $3 \cdot 115696$ & $6 \cdot 090825$ & $8 \cdot 841488$ & $11 \cdot 34631$ & $13 \cdot 62079$ & $15 \cdot 69385$ \\
18 & $3 \cdot 115696$ & $6 \cdot 090825$ & $8 \cdot 841462$ & $11 \cdot 34630$ & $13 \cdot 62074$ & $15 \cdot 69364$ \\
\hline
\end{tabular}

conditions are given in tables 2 to 5 for the different thickness-to-length ratios. The frequencies obtained are compared with the previously published results of CBT (Hurty \& Rubinstein 1967) and FSDT (Lee \& Schultz 2004; Kocatürk \& Şimşek 2005a). Convergence study of the beam with pinned-pinned boundary conditions is carried out for $h / L=0 \cdot 1$ and the results are given in table 1. In all the following calculations, Poisson's ratio is taken as $v=0.3$ and thickness-to-length ratios range from $h / L=0.002$ to $0 \cdot 2$.

It is observed from table 1 that the natural frequencies decrease as the number of polynomial terms increases. It means that the convergence to the exact value is from above, i.e. by increasing the number of the polynomial terms, the exact value can be approached from above. It should be remembered that energy methods always overestimate the fundamental frequency, so with more refined analyses, the exact value can be approached from above. From here on, the number of the polynomial terms $N$ is taken as 16 in all of the numerical investigations.

It is known that for simplifying some problems such as beam problems and plate problems, some restrictions are made and some unknown functions are expressed by other unknown

Table 2. The first six dimensionless frequency parameters $\lambda_{i}$ of the clamped-clamped (CC) beam for different $h / L$ values.

\begin{tabular}{|c|c|c|c|c|c|c|c|}
\hline Method & $h / L$ & $\lambda_{1}$ & $\lambda_{2}$ & $\lambda_{3}$ & $\lambda_{4}$ & $\lambda_{5}$ & $\lambda_{6}$ \\
\hline $\mathrm{CBT}^{1}$ & & $4 \cdot 7300$ & $7 \cdot 8532$ & 10.9956 & $14 \cdot 1372$ & $17 \cdot 2788$ & $20 \cdot 4204$ \\
\hline FSDT $^{2}$ & & 4.7299 & $7 \cdot 8529$ & 10.9949 & $14 \cdot 1358$ & 17.2765 & $20 \cdot 4166$ \\
\hline TSDT $^{3}$ & & 4.7299 & $7 \cdot 8529$ & 10.9949 & 14.1359 & 17.2766 & $20 \cdot 4170$ \\
\hline FSDT & & 4.7296 & $7 \cdot 8516$ & 10.9916 & $14 \cdot 1$ & $17 \cdot 2650$ & $20 \cdot 3983$ \\
\hline TSDT & & 4.7296 & $7 \cdot 8516$ & $10 \cdot 9$ & & 17. & $20 \cdot 3989$ \\
\hline FSDT & & $4 \cdot 7283$ & $7 \cdot 8468$ & 10.9799 & $14 \cdot 1$ & 17.2244 & $20 \cdot 3336$ \\
\hline TSDT & & 4.7284 & $7 \cdot 8469$ & 10.98 & 14. & $17 \cdot 2$ & $20 \cdot 3350$ \\
\hline FSDT & & $4 \cdot 72$ & $7 \cdot 82$ & $10 \cdot 9$ & 14. & & $20 \cdot 0866$ \\
\hline TSDT & 0 & 4.7235 & $7 \cdot 8283$ & 10.9345 & $14 \cdot 0$ & $17 \cdot($ & $20 \cdot 0911$ \\
\hline FSDT & & 4.6898 & $7 \cdot 7035$ & 10.6399 & $13 \cdot 4$ & 16. & 18.7316 \\
\hline SDT & & $4 \cdot 6$ & $7 \cdot 70$ & & & & \\
\hline FSDT & & 4.5795 & $7 \cdot 33$ & 9.85 & $12 \cdot 1$ & 14.2 & $16 \cdot 1478$ \\
\hline TSDT & 0 & 4.5820 & $7 \cdot 3407$ & $9 \cdot 8810$ & $12 \cdot 1$ & 14.3018 & $16 \cdot 2373$ \\
\hline $\mathrm{T}$ & & $4 \cdot 22$ & $6 \cdot 4$ & & & & \\
\hline TSDT & 0 & $4 \cdot 2563$ & $6 \cdot 4642$ & $8 \cdot 3758$ & $10 \cdot 0364$ & 11.5314 & $12 \cdot 8563$ \\
\hline
\end{tabular}

$\left({ }^{1}\right.$ Hurty \& Rubinstein $1967 ;{ }^{2}$ Kocatürk \& Şimşek 2005a; ${ }^{3}$ present study) 
Table 3. The first six dimensionless frequency parameters $\lambda_{i}$ of the clamped-pinned (CP) beam for different $h / L$ values.

\begin{tabular}{|c|c|c|c|c|c|c|c|}
\hline Method & $h / L$ & $\lambda_{1}$ & $\lambda_{2}$ & $\lambda_{3}$ & $\lambda_{4}$ & $\lambda_{5}$ & $\lambda_{6}$ \\
\hline $\mathrm{CBT}^{1}$ & & 3.9269 & $7 \cdot 0685$ & $10 \cdot 2101$ & $13 \cdot 3517$ & $16 \cdot 4933$ & 19.6349 \\
\hline FSDT $^{2}$ & & 3.9265 & 7.0684 & $10 \cdot 2097$ & $13 \cdot 3508$ & $16 \cdot 4916$ & 19.6319 \\
\hline TSDT $^{3}$ & $0 \cdot 002$ & 3.9265 & 7.0684 & $10 \cdot 2097$ & $13 \cdot 3508$ & $16 \cdot 4916$ & 19.6322 \\
\hline FSDT & & 3.9264 & $7 \cdot 0676$ & $10 \cdot 2074$ & $13 \cdot 3458$ & $16 \cdot 4825$ & 19.6169 \\
\hline TSDT & 0.00 & 3.9264 & 7.0676 & 10 . & 13 & 26 & 19.6173 \\
\hline FSDT & & 3.9258 & $7 \cdot 0646$ & $10 \cdot 1$ & 13. & 16 . & 19.5638 \\
\hline TSDT & $0 \cdot 0$ & 3.9258 & $7 \cdot 06$ & $10 \cdot 1$ & 13. & 16 . & 19.5646 \\
\hline FSDT & & 3.9234 & $7 \cdot 05$ & $10 \cdot 1$ & 13 . & 16 . & $19 \cdot 3601$ \\
\hline TSDT & 0 & 3.9234 & $7 \cdot 0531$ & $10 \cdot 1$ & $13 \cdot 2$ & 16 . & $19 \cdot 3624$ \\
\hline FSDT & & 3.9071 & $6 \cdot 97$ & & & & $18 \cdot 2150$ \\
\hline TSDT & & 3.9072 & 6.97 & 00 & 12 & 15 & $18 \cdot 2290$ \\
\hline FSDT & & 3.8517 & 6.7305 & $9 \cdot 3658$ & 11.7583 & 13.9329 & 15.9194 \\
\hline TSDT & 0 . & 3.8525 & $6 \cdot 7346$ & & 11.78 & & 15.9742 \\
\hline FSDT & & 3.66 & $6 \cdot 07$ & 807 & & & $12 \cdot 6191$ \\
\hline TSDT & $0 \cdot$ & 3.6708 & 6.0947 & $8 \cdot 1219$ & 9.8636 & $11 \cdot 3979$ & 12.7717 \\
\hline
\end{tabular}

$\left({ }^{1}\right.$ Hurty \& Rubinstein $1967 ;{ }^{2}$ Kocatürk \& Şimşek 2005a; ${ }^{3}$ present study)

functions. This situation results in decreasing the freedom of the considered problem. As is known, the frequencies become greater when the considered element becomes more rigid. Therefore, by decreasing the freedom of the element, the frequencies become greater from the exact frequencies. In CBT, the plane cross sections remain plane and perpendicular to the elastic curve after bending. In this case, rotations of the cross sections of the beam are expressed in terms of displacements. The first derivative of the elastic curve of the beam with

Table 4. The first six dimensionless frequency parameters $\lambda_{i}$ of the pinned-pinned (PP) beam for different $h / L$ values.

\begin{tabular}{|c|c|c|c|c|c|c|c|}
\hline Method & $h / L$ & $\lambda_{1}$ & $\lambda_{2}$ & $\lambda_{3}$ & $\lambda_{4}$ & $\lambda_{5}$ & $\lambda_{6}$ \\
\hline $\mathrm{BT}^{1}$ & & $3 \cdot 1415$ & $6 \cdot 2831$ & 9.4247 & $12 \cdot 5664$ & $15 \cdot 7080$ & $18 \cdot 8496$ \\
\hline FSDT $^{2}$ & & $3 \cdot 1415$ & $6 \cdot 2831$ & 9.4244 & $12 \cdot 5656$ & $15 \cdot 7066$ & $18 \cdot 8471$ \\
\hline TSDT $^{3}$ & 0.002 & $3 \cdot 1415$ & $6 \cdot 283$ & 9.4244 & $12 \cdot 5$ & $15 \cdot 7066$ & $18 \cdot 8472$ \\
\hline FSDT & & $3 \cdot 1415$ & $6 \cdot 2$ & $9 \cdot 4$ & 12 & 15 & 351 \\
\hline TSDT & & $3 \cdot 1415$ & $6 \cdot 2826$ & $9 \cdot 4229$ & $12 \cdot 5$ & 96 & $18 \cdot 8352$ \\
\hline FSDT & & $3 \cdot 1413$ & $6 \cdot 28$ & 9.4176 & $12 \cdot$ & & 18.7925 \\
\hline TSDT & $0 \cdot($ & $3 \cdot 1413$ & $6 \cdot 2810$ & 9.4176 & $12 \cdot 5$ & 15.6749 & 18.7926 \\
\hline FSDT & & $3 \cdot 1405$ & $6 \cdot 2$ & $9 \cdot 3962$ & $12 \cdot 4$ & $15 \cdot 5$ & 18.6280 \\
\hline TSDT & & $3 \cdot 1$ & $6 \cdot 2$ & $9 \cdot 3$ & 12 & 15 & 18.6283 \\
\hline FSDT & & $3 \cdot 1349$ & $6 \cdot 2313$ & 9.2553 & $12 \cdot 1$ & 14.9926 & $17 \cdot 6802$ \\
\hline TSDT & $0 \cdot 0$ & $3 \cdot 1349$ & $6 \cdot 2313$ & $9 \cdot 25$ & $12 \cdot 1$ & 14.99 & 17.6829 \\
\hline FSDT & & $3 \cdot 1$ & $6 \cdot 0$ & 8. & & & 15.6769 \\
\hline TSDT & 0 & $3 \cdot 11$ & 6.0908 & $8 \cdot 8414$ & $11 \cdot 3$ & $13 \cdot 6207$ & 15.6938 \\
\hline FSDT & & $3 \cdot 04$ & & & & & 12.5971 \\
\hline TSDT & 0 & 3.0454 & 5.6731 & 7.8469 & 9.6769 & $11 \cdot 2625$ & 12.6723 \\
\hline
\end{tabular}

( ${ }^{1}$ Hurty \& Rubinstein $1967 ;{ }^{2}$ Kocatürk \& Şimşek 2005a; ${ }^{3}$ present study) 
Table 5. The first six dimensionless frequency parameters $\lambda_{i}$ of the pinned-guided (PG) beam for different $h / L$ values.

\begin{tabular}{|c|c|c|c|c|c|c|c|}
\hline Method & $h / L$ & $\lambda_{1}$ & $\lambda_{2}$ & $\lambda_{3}$ & $\lambda_{4}$ & $\lambda_{5}$ & $\lambda_{6}$ \\
\hline $\mathrm{CBT}^{1}$ & & 1.5708 & 4.7123 & 7.8539 & $10 \cdot 9955$ & $14 \cdot 1371$ & $17 \cdot 2787$ \\
\hline FSDT $^{4}$ & & 1.5708 & $4 \cdot 7123$ & $7 \cdot 8538$ & $10 \cdot 9951$ & $14 \cdot 1362$ & $17 \cdot 2770$ \\
\hline $\mathrm{TSDT}^{3}$ & & 1.5708 & 4.7123 & $7 \cdot 8538$ & $10 \cdot 9951$ & $14 \cdot 1362$ & $17 \cdot 2770$ \\
\hline FSDT & & $1 \cdot 5708$ & $4 \cdot 7121$ & $7 \cdot 8529$ & & 14 . & $17 \cdot 2677$ \\
\hline TSDT & & 1.5708 & $4 \cdot 7121$ & 7.8529 & 10.9927 & $14 \cdot 1311$ & $17 \cdot 2677$ \\
\hline FSDT & & $1 \cdot 5707$ & 4.7114 & $7 \cdot 8498$ & $10 \cdot 9842$ & $14 \cdot 1130$ & $17 \cdot 2348$ \\
\hline TSDT & $0 \cdot 0$ & $1 \cdot 5707$ & $4 \cdot 7114$ & $7 \cdot 8498$ & $10 \cdot 9842$ & $14 \cdot 1131$ & $17 \cdot 2349$ \\
\hline FSDT & & 1.5706 & $4 \cdot 7088$ & 7.8374 & $10 \cdot 9505$ & 14.0423 & $17 \cdot 1073$ \\
\hline TSDT & & 1.5706 & 4.7088 & $7 \cdot 8375$ & $10 \cdot 9505$ & $14 \cdot 0423$ & $17 \cdot 1073$ \\
\hline FSDT & & 1.5699 & 4.6902 & $7 \cdot 7542$ & $10 \cdot 7319$ & $13 \cdot 6020$ & $16 \cdot 3524$ \\
\hline TSDT & $0 \cdot 05$ & 1.5699 & 4.6903 & $7 \cdot 7542$ & $10 \cdot 7320$ & $13 \cdot 6025$ & $16 \cdot 3537$ \\
\hline FSDT & & 1.5674 & 4.6276 & 7.4963 & $10 \cdot 1223$ & $12 \cdot 5056$ & 14.6697 \\
\hline TSDT & 0 & $1 \cdot 5675$ & 4.6277 & 7.4967 & $10 \cdot 1241$ & $12 \cdot 5106$ & 14.6805 \\
\hline FSDT & & $1 \cdot 5578$ & 4.4202 & $6 \cdot 8065$ & 8.7852 & $10 \cdot 4663$ & 11.9320 \\
\hline TSDT & 0.2 & $1 \cdot 5578$ & 4.4207 & $6 \cdot 8103$ & 8.7979 & $10 \cdot 4953$ & 11.9861 \\
\hline
\end{tabular}

$\left({ }^{4}\right.$ Lee \& Schultz 2004)

respect to the coordinate along the axis of the beam gives the rotation function. In FSDT, plane sections remain plane but not necessarily perpendicular to the elastic curve after bending. In TSDT, plane sections are not plane and are not perpendicular to the elastic curve in general cases. In these theories, TSDT satisfies the free surface stress conditions. At the upper and lower surfaces of the beam, the shear stresses are zero in the third-order shear deformation theory. It can be deduced from these explanations that in these three categories of beam theories, frequencies of TSDT should be lower than those of others. However, it is interesting to note that this is not so because it can be deduced from tables 2 to 5 that the frequencies of TSDT remain between the frequencies of CBT and FSDT. This situation can be explained as follows. The displacement field $u_{x}$ for the first-order shear deformation theory (FSDT) is $u_{x}=z \phi^{\mathrm{FSDT}}$ (Wang et al 2000), and for the third-order shear deformation theory (TSDT) $u_{x}=z \phi^{\mathrm{TSDT}}-\alpha z^{3}\left(\phi^{\mathrm{TSDT}}+w_{, x}\right)$ in equation (1). It is expected that the rotations $\phi^{F S D T}$ and $\phi^{\text {TSDT }}$ must be very close to each other. In this case, because of the negative terms in the displacement equation of TSDT, the displacements of TSDT become generally smaller than the displacements of FSDT. As a result of this situation, the strains of TSDT become generally smaller than the strains of FSDT. Therefore, the more flexible FSDT scheme results in greater displacements and smaller frequencies compared to the TSDT scheme. A similar situation is encountered in the frequency tables given for the classical plate, the first-order plate and the third-order plate theories (Reddy 1984).

The three solutions are close to each other for small values of $h / L$ (i.e. $h / L=0.002$ and 0.005 ) as seen from tables 2 to 5 . The results of TSDT are a little greater than those of FSDT.

It should be remembered that the eigenvalues obtained using the first-order or higher-order beam theories are lower than the corresponding eigenvalues obtained by the classical beam theory. The FSDT and TSDT results are very close to each other for the considered parameters. It can be seen from tables 2 to 5 that the results obtained using FSDT are fairly accurate; the difference between the results of FSDT and TSDT increases with increasing mode number. 
Although the TSDT results are more accurate than the CBT and FSDT results for beams with rectangular cross-sections, higher-order beam theories cannot be used for beams with cross-sections other than rectangular shape.

It can be deduced from tables 2 to 5 that the difference between the eigenvalues of CBT and the other two theories increases for increasing mode numbers. This means that the effect of shear deformations increases for increasing mode numbers.

Tables 2 to 5 also show that the difference between the eigenvalues of CBT and the other two theories increases for increasing thickness-to-length ratios $h / L$. The effect of shear deformations increases for increasing values of $h / L$.

\section{Conclusions}

The free vibrations of the beams have been investigated for different thickness-to-length ratios according to TSDT. The eigenvalues of the beams obtained with various boundary conditions are compared with the previously available results of CBT and FSDT. Using Lagrange's equations with the trial functions in the polynomial form and satisfying the constraint conditions by the use of Lagrange multipliers is a nice way for studying the free vibration characteristics of the beams.

Numerical calculations have been carried out to clarify the effects of the thickness-to-length ratio on the eigenvalues of the beams. It is observed from the investigations that the CBT, FSDT and TSDT results are close to each other for small values of $h / L$. However, as the thicknessto-length ratio becomes greater, the results of the classical beam theory significantly differ from others. This situation is also observed as the mode numbers increase. It is interesting to note that the frequencies of TSDT are slightly greater than that of FSDT. Although it is not investigated here, it is expected that the results of the third-order shear deformation theory give the closest frequency values to the exact frequency values in the considered three beam theories as it is proved for plates (Reddy 1984). The results obtained are accurate and are expected to be useful to other researchers for comparison.

\section{Appendix}

$$
\begin{aligned}
& \tilde{K}_{(m)(n)}=\frac{16}{5} \kappa \int_{-1 / 2}^{1 / 2}\left(x^{m-1}\right)^{\prime}\left(x^{n-1}\right)^{\prime} \mathrm{d} x+\frac{1}{21} \int_{-1 / 2}^{1 / 2}\left(x^{m-1}\right)^{\prime \prime}\left(x^{n-1}\right)^{\prime \prime} \mathrm{d} x m, n=1,2, \ldots, N \\
& \tilde{K}_{(m)(N+n)}=\frac{16}{5} \kappa \int_{-1 / 2}^{1 / 2}\left(x^{m-1}\right)\left(x^{n-1}\right)^{\prime} \mathrm{d} x-\frac{16}{105} \int_{-1 / 2}^{1 / 2}\left(x^{m-1}\right)^{\prime}\left(x^{n-1}\right)^{\prime \prime} \mathrm{d} x \\
& \tilde{K}_{(N+m)(n)}=\frac{16}{5} \kappa \int_{-1 / 2}^{1 / 2}\left(x^{m-1}\right)^{\prime}\left(x^{n-1}\right) \mathrm{d} x-\frac{16}{105} \int_{-1 / 2}^{1 / 2}\left(x^{m-1}\right)^{\prime \prime}\left(x^{n-1}\right)^{\prime} \mathrm{d} x \\
& m, n=1,2, \ldots, N \\
&
\end{aligned}
$$




$$
\begin{aligned}
& \tilde{K}_{(N+m)(N+n)}=\frac{16}{5} \kappa \int_{-1 / 2}^{1 / 2}\left(x^{m-1}\right)\left(x^{n-1}\right) \mathrm{d} x+\frac{68}{105} \int_{-1 / 2}^{1 / 2}\left(x^{m-1}\right)^{\prime}\left(x^{n-1}\right)^{\prime} \mathrm{d} x \\
& m, n=1,2, \ldots, N \\
& \tilde{M}_{(m)(n)} \quad=\int_{-1 / 2}^{1 / 2}\left(x^{m-1}\right)\left(x^{n-1}\right) \mathrm{d} x+\frac{\mu}{252} \int_{-1 / 2}^{1 / 2}\left(x^{m-1}\right)^{\prime}\left(x^{n-1}\right)^{\prime} \mathrm{d} x \\
& m, n=1,2, \ldots, N \\
& \tilde{M}_{(m)(N+n)} \quad=\left(\frac{1}{252}-\frac{1}{60}\right) \mu \int_{-1 / 2}^{1 / 2}\left(x^{m-1}\right)\left(x^{n-1}\right)^{\prime} \mathrm{d} x \quad m, n=1,2, \ldots, N \\
& \tilde{M}_{(N+m)(n)} \quad=\left(\frac{1}{252}-\frac{1}{60}\right) \mu \int_{-1 / 2}^{1 / 2}\left(x^{m-1}\right)^{\prime}\left(x^{n-1}\right) \mathrm{d} x \quad m, n=1,2, \ldots, N \\
& \tilde{M}_{(N+m)(N+n)}=\left(\frac{1}{12}+\frac{1}{252}-\frac{1}{30}\right) \mu \int_{-1 / 2}^{1 / 2}\left(x^{m-1}\right)\left(x^{n-1}\right) \mathrm{d} x \\
& m, n=1,2, \ldots, N
\end{aligned}
$$

The elements of matrices $\tilde{K}$ and $\tilde{M}$ are obtained from the boundary conditions (Lagrange multipliers) are not given here.

\section{List of symbols}

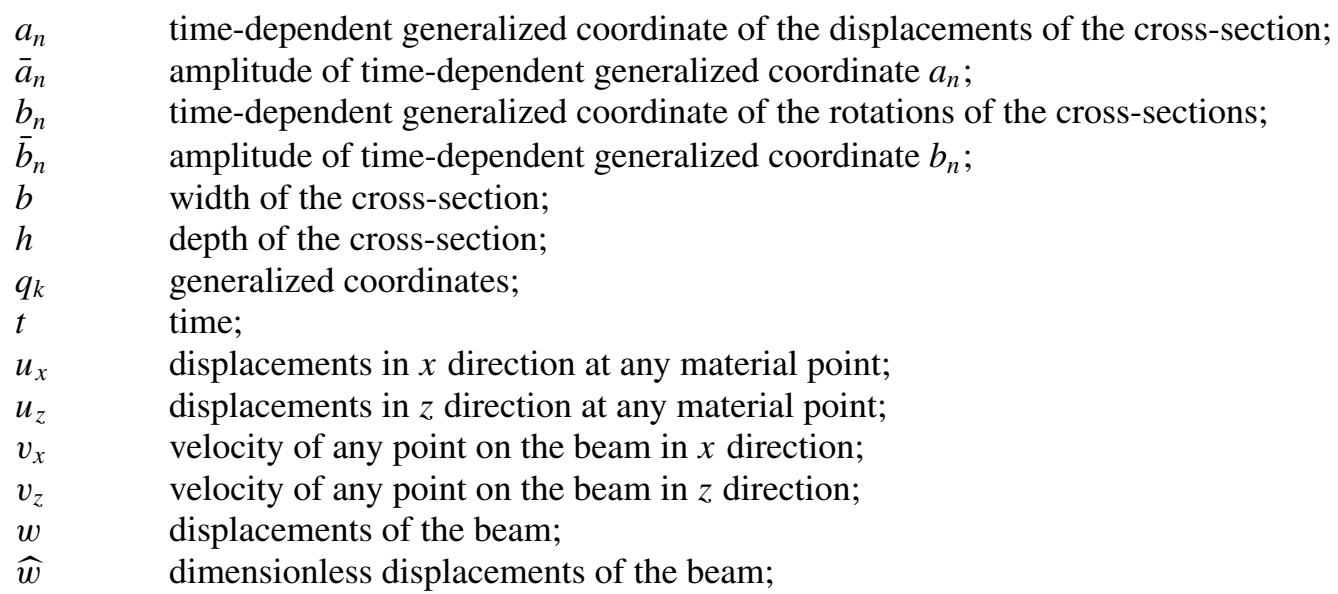


$\bar{w} \quad$ dimensionless amplitudes of the displacements;

$x \quad x$ coordinate;

$\hat{x} \quad$ dimensionless $x$ coordinate;

$y \quad y$ coordinate;

$z \quad z$ coordinate;

$A_{x z} \quad$ a cross-sectional stiffness coefficients;

$D_{x x} \quad$ a cross-sectional stiffness coefficients;

$D_{x z} \quad$ a cross-sectional stiffness coefficients;

$E \quad$ elastic modulus of the beam;

$F_{x x} \quad$ a cross-sectional stiffness coefficients;

$F_{x z} \quad$ a cross-sectional stiffness coefficients;

$G \quad$ shear modulus of the beam;

$G_{S} \quad$ Lagrange multipliers formulation;

$H_{x x} \quad$ a cross-sectional stiffness coefficients;

$I_{A} \quad$ a cross-sectional inertial coefficients;

$I_{D} \quad$ a cross-sectional inertial coefficients;

$I_{F} \quad$ a cross-sectional inertial coefficients;

$I_{H} \quad$ a cross-sectional inertial coefficients;

$\tilde{K} \quad$ stiffness matrix of the beam;

$L \quad$ length of the beam;

$M \quad$ number of the Lagrange multipliers;

$\tilde{M} \quad$ mass matrix of the beam;

$M_{x x} \quad$ bending moment;

$N \quad$ number of the polynomial terms;

$P_{x x} \quad$ a higher order stress resultants;

$Q_{x} \quad$ shear force;

$R_{x} \quad$ a higher order stress resultants;

$T \quad$ kinetic energy of the beam;

$V \quad$ strain energy of the beam;

$\widehat{V}_{x} \quad$ effective shear force;

$\alpha \quad$ a parameter in the displacement fields (equal to $4 / 3 h^{2}$ );

$\beta_{1}, \beta_{2} \quad$ Lagrange multipliers;

$\delta_{1}, \delta_{2} \quad$ Lagrange multipliers;

$\varepsilon_{x x} \quad$ longitudinal normal strain;

$\phi \quad$ rotations of the cross-sections;

$\widehat{\phi} \quad$ dimensionless rotations of the cross-sections;

$\bar{\phi} \quad$ dimensionless amplitudes of the rotations of the cross-sections;

$\gamma_{x z} \quad$ transverse shear strain;

$\kappa \quad$ a dimensionless quantity;

$\lambda_{1}, \lambda_{2}, \ldots$ dimensionless frequency parameter of the beam;

$\mu \quad$ a dimensionless quantity;

$v \quad$ Poisson's ratio;

$\theta_{1}, \theta_{2} \quad$ Lagrange multipliers;

$\rho \quad$ mass of the beam per unit volume;

$\sigma_{x x} \quad$ longitudinal normal stress;

$\tau_{x z} \quad$ transverse shear stress;

$\omega \quad$ natural frequency of the beam. 


\section{References}

Auciello N M, Ercolano A 2004 A general solution for dynamic response of axially loaded nonuniform Timoshenko beams. Int. J. Solids and Struct. 41: 4861-4874

Banerjee J R 1998 Free vibration of axially loaded composite Timoshenko beams using the dynamic stiffness matrix method. Comput. Struct. 69: 197-208

Eisenberger M 2003 An exact high order beam element. Comput. Struct. 81: 147-152

Farghaly S H 1994 Vibration and stability analysis Timoshenko beams with discontinuities in crosssection. J. Sound Vib. 174: 591-605

Hurty W C, Rubinstein M F 1967 Dynamics of Structures (New Delhi: Prentice Hall)

Khdeir A A, Reddy J N 1997 An exact solution for the bending of thin and thick cross-ply laminated beams. Comput. Struct. 37: 195-203

Kim H K, Kim M S 2001 Vibration of beams with generally restrained boundary conditions using Fourier series. J. Sound Vib. 245(5): 771-784

Kocatürk T, Şimşek M 2005a Free vibration analysis of Timoshenko beams under various boundary conditions. Sigma J. Eng. and Nat. Sci. 2005/1: 108-122

Kocatürk T, Şimşek M 2005b Free vibration analysis of elastically supported Timoshenko beams. Sigma J. Eng. and Nat. Sci. 2005/3: 79-93

Lee J, Schultz W W 2004 Eigenvalue analysis of Timoshenko beams and axisymmetric Mindlin plates by the pseudospectral method. J. Sound Vib. 269: 609-621

Lee Y Y, Wang C M, Kitipornchai S 2003 Vibration of Timoshenko beams with internal hinge. J. Eng. Mech. 2003/3: 293-301

Nallim L G, Grossi R O 1999 A general algorithm for the study of the dynamical behaviour of beams. Appl. Acoustic 57: 345-356

Reddy J N 1984 Energy and variational methods in applied mechanics. (John Wiley \& Sons, Inc. NY)

Soldatos K P, Sophocleous C 2001 On shear deformable beam theories: The frequency and normal mode equations of the homogeneous orthotropic Bickford beam. J. Sound Vib. 242: 215-245

Şimşek M 2005a Free vibration analysis of beams subjected to axial load under various boundary conditions. Sigma J. Eng. and Nat. Sci. 2005/3: 1-10

Şimşek M 2005b Investigation of the behaviour of the beams subjected to an eccentric compressive force and a moving harmonic force. Phd. Dissertation report. Yildiz Technical University (Istanbul)

Timoshenko S, Young D H 1955 Vibration Problems in Engineering. (New York: Van Nostrand Company)

Wang C M, Reddy J N, Lee K H 2000 Shear Deformable Beams and Plates. (Amsterdam: Elsevier Science Ltd)

Zhou D 2001 Free vibration of multi-span Timoshenko beams using static Timoshenko beam functions. J. Sound Vib. 241: 725-734 